The circumference of the head through the occipitomental diameter measured $27 \frac{1}{2}$ inches; from the angle of the face to the symphysis, $4_{4}^{3}$ inches; circumference of hand around the palm, $10 \frac{1}{4}$ inches; width of palm, $4 \frac{1}{2}$ inches.

The costo-cartilages were completely calcified so that it was necessary to saw them in two in order to open the thorax. The lungs were sound with calcified points, size of a millet-seed on the surface. The heart was hypertrophied, but no evidence of valvular disease. There was no calcareous deposit in the endocardial region. The right ventricle was filled with blood; the left partially so. Heart stopped in diastole. Postmortem and antemortem clots had formed in both ventricles. No disease of the heart was discernible. The mucous membrane of the stomach was highly congested, and enlarged blood vessels roughened its surface. This condition is most likely accounted for by the long absence of food. A small upper portion of the ileum was filled with fecal matter of considerable consistence; the lower part was filled with gas. The large intestine was normal, except an occasional enlarged gland undergoing cheesy degeneration, probably antecedent to calcification. Appendix vermiformis was normal.

An indenture on the external surface of the calvarium, posterior to the coronal suture on right side, indicated that at some time the skull had received a blow from some blunt instrument. No history, however, of an injury having been received was obtained. The internal surface of the skull was roughened by a number-a dozen or more or less-of sharp-pointed spinous projections, varying from one-sixteenth to one-eight of an inch in length, which pressed hard upon the dura mater.

The upper surface of the hemispheres of the brain was apparently normal. The base, however, showed an imperforate and knotted condition of the posterior cerebral artery on the left side. It looked like a string of beads. The remaining portion of the surface of the base was normal, except in the region of the sella turcica, where the pituitary body was greatly enlarged. This glandular structure had grown to the dimensions of a medium-sized hen's egg. The dura mater round and about it was calcified, and it was difficult to remove the body from its attachments. The average weight of this body is from 5 to 10 grains, but this one weighed, after having lain in alcohol six months, 476 grains.

The questions that naturally arise are: Did the growth of this gland cause the peculiar enlargement of the face, hands and feet? What caused the obstinate vomiting, and what produced the Cheyne-Stokes respiration?

SEPTIC DISEASES OF THE KNEE JOINT. Read before the Wabasha County Medical Society.

$$
\begin{aligned}
& \text { HY C. H. MAYO, M.D. } \\
& \text { SURGEON TO ST. MARY'S HOSPITAL, } \\
& \text { ROCHESTER, MINN. }
\end{aligned}
$$

The great size and peculiar formation of the knee joint, with its many communicating bursæ, render its destruction probable by infection, and the loss of the limb, or life itself, is of comparative frequency.

A brief review of the more important of its anatomic features will explain why sepsis is so destructive.

The strength of this largest articulation of the body is in its powerful ligaments, which unite its opposing surfaces, articulating only by contact. The bony axis of the limb changes at the knee, the femur slanting inward from above and the tibia being vertical. The lateral ligaments of the joint have the least strength. The posterior are strong and the capsule becomes ligamentous anteriorly. The most powerful and important ligaments of the joint are the crucial. The synovial membrane extends above the patella and beneath the extensor tendon, the cul-de-sac reaching a point one inch or more above the trochlear surface of the femur. Above the synovial sac is a bursa about one inch long separating the quadriceps tendon from the femur and communicating with the joint in four out of five cases. The upper third of the ligamentum patellæ is separated from the synovial sac by a pad of fat, the lower two-thirds are in relation to the bursa interposed between it and the tubercle of the tibia. In the popliteal space interposed between the internal condyle of the femur, the inner head of the gastrocnemius and the semimembranosus is another bursa usually communicating with the knee joint. The bursa beneath the popliteus tendon always connects with the joint and usually with the tibio-fibular articulation. The posterior part of the synovial sac has other bursal pouches in the adult. The epiphyseal line of the femur is intracapsular, showing the route of infection in the complicating joint abscess, occurring in some cases of periosteal and osteomyelitic diseases, and the source of effusion in fractures involving this region.

In a consideration of septic diseases of the knee joint we are only concerned in the more simple injuries causing synovitis, when auto-infection renders them septic. The entrance of blood into the joint is of the same significance, as a slight injury may occasion a hemorrhage, which never entirely coagulates, regardless of the quantity effused, but mixes with the synovia, and its absorption is extremely slow. These cases, through delay in absorption, or improper management, may develop the simple purulent synovitis.

Acute purulent synovitis occurs in young children, as a rule, in the first few weeks of life, and has been considered a probable umbilical infection. These cases discharge early and in most cases the joint function is not permanently impaired - as shown by Krause and Volkmann. In a case of doubt concerning the character of a joint effusion the hypodermic can be used for aspiration with the same freedom as it is now used on the chest to demonstrate fluids in the pleura. The withdrawal of a small quantity of serous effusion has the same effect as is so frequently noticed in the pleura, the stimulation of absorption. In the more simple purulent collections, a large caliber hypodermic needle can be used to withdraw the contents of the joint, and then distend it with a 1 to 1000 bichlorid solution. After a massage of the joint withdraw the bichlorid solution, repeating the process with normal salt solution.

Gonorrheal synovitis, which has been shown by Councilman and Ott to be due to auto-infection by the gonococcus, may be treated in the same manner, after a thorough trial of large doses of potassium iodid has been made without relief.

Penetrating wounds of the knee joint, including incised, punctured, lacerated and gunshot injuries, are well classed among the most dangerous of bodily injuries not proving immediately fatal. Their gravity depends solely upon the introduction of septic matter into a serous cavity at the time of injury, or during its subsequent care. 
The simpler forms of lacerated and punctured wounds are the most frequent, as distinguished from the complicated cases in which the injuries to adjacent structures may be of more serious consequence than the opening of the joint. Where the wound is small and the synovial sac not visible great care must be exercised in judging of joint penetration. The free flow of synovial fluid may only indicate the opening of a bursa, or large tendon sheath; the rapid distension of the joint with blood may also come from a contusion, without joint penetration.

In cases in which the nature of the injury would indicate a possible foreign body, septic origin or contamination, the probable diagnosis must be confirmed by exploration of the wound, under strict antiseptic precautions, when, should the synovial sac be found opened, the interior of the joint should be thoroughly flushed with a 1 to 1000 bichlorid solution and drainage established, preferably by means of folded rubber tissue, and a copious dressing applied. In cases of joint injury where we can be reasonably certain of an aseptic wound, an antiseptic dressing may be applied, with rest of joint, awaiting the development of symptoms before further interference.

Should infection occur during treatment, the incision should be enlarged, antiseptic irrigation applied and freer drainage established. Synovia may flow for several days, and unless infection supervene, a perfect recovery results. In those cases in which infection occurs the result obtained will depend upon several factors, viz.: The nature and degree of infection, the freedom of drainage and the treatment applied. Infection produced by the less virulent pus-producing germs may not be clinically demonstrable for from two to seven days. The turbid effusion filled with flakes of lymph will then indicate vascularization of the synovial sac. The joint becomes red, swollen, painful and tender; a chill more or less severe indicates the commencement of infection.

Systemic toxemia is evidenced by a fever, which continues as long as the joint is distended with pus. In the less virulent infection, if a free drainage is secured, the joint structures may be maintained and a fair degree of joint function preserved, even after several weeks of discharge. Insufficient drainage, or a more virulent form of infection, may rapidly so disintegrate the joint structures that a functional result is impossible. The ligaments become softened, the cartilages necrosed and separated, the synovial membrane thickened and the extension of the purulent process into adjacent bursæ and tendon sheaths develops abscesses in the thigh and posterior tibial regions. The limb is enormously swollen and the general septic condition is very marked. Life may be saved and recovery hastened by an early amputation, otherwise death may ensue from toxemia, or after months of discharge life and limb may be saved, with a functionless joint.

In the case of streptococcus infection, the course is more rapid and death frequently occurs early, from phlebitis, gangrene or septicemia. In mild or mixed infections secondary to chronic joint disease it is possible to have a walling off of the abscess in a portion of the synovial pouch, as mentioned by Andrews, similar to like conditions in the peritoneum or pleura. Gunshot wounds of this joint require the same care as the lacerated and punctured wounds. Injuries by large missiles require exploration, and in case of serious bone injury a partial arthrectomy may be necessary, while those made by small caliber bullets may be treated as doubtful cases until interference is demanded by the symptoms developed.

Bullets are usually sterile, but there is always danger from the particles of clothing carried in by them. In gunshot wounds of this joint, unless serious hemorrhage occur requiring control, the first consideration is the application of an antiseptic dressing. Dividing the knee-joint cavity into spaces and treating suppuration by drainage with four to six drainage tubes, as recommended by Andrews and Gerster, suffice for some cases, and may be supplemented by a tube drain through the popliteal space, as practiced by Hartley. Edmond Owen recommends the vertical incision on either side of the patella, and in some cases to connect the two incisions by cutting the patella ligament.

Frederic S. Dennis and Roswell Park report cases successfully treated by continuous irrigation or immersions in weak antiseptic fluids. The one essential feature in the treatment of the more serious forms of phlegmonous inflammation of the joint is free drainage. The transverse incision from prominence to prominence of the condyles across the front of the joint, as made for resection, presents the freer exposure for dry gauze pack of all parts of the joint, or the use of the gauze pack saturated with a solution of 5 per cent. balsam Peru in castor oil, so successfully employed by W. W. Van Arsdale in the treatment of suppurating wounds. This incision will accomplish all that an amputation can promise, with less shock and with the saving of a useful limb, and in many cases a limited degree of motion.

In three cases in which the patella was severed through its center, the two halves afforded a firm hold for passing sutures behind and closing the joint at a later period.

In the January, 1895, issue of the Annals of Surgery, is reported my first case treated in this manner and the successful treatment of four similar cases in the past two years, leads me to emphasize my former statements. Two of these cases have one-fourth motion. In the Annals of Surgery, Vol. XXII, page 503 , in report of transactions of the New York Surgical Section, is the following from Dr. Arpad Gerster, which so perfectly describes the treatment, I present part of it here: "Dr. Mayo's suggestion struck him as very plausible, and he has applied it in two cases. The result had been excellent. As all know, these acute forms of joint phlegmon necessarily end in ankylosis, if, indeed, they do not cause loss of limb or life. The cartilaginous covering of the bones forming the joint is destroyed, and therefore ankylosis is inevitable. Knowing this fact, the surgeon in adopting this method of treatment frankly accepts the necessity for ankylosis. The road then becames very clear. The treatment consists in laying the joint open by a transverse incision, extending from one condyle to the other, just as a joint is laid open for resection. Then as many more incisions are made as may be necessary, in order to open up every collection of pus or fluid in or about the joint. The capsule in all these cases is very intumescent and swollen, and for this reason occludes all drainage tubes in a way to make retention inevitable. Then lay the joint freely open, removing or not removing tissues, as the case may be, pack and drain every recess, and treat it as an open wound. It is astonishing how rapidly the phleg- 
monous process ceases, when compared with other methods of treatment. After the symptoms of active inflammation have subsided, the joint is put in proper position and ankylosis is allowed to take place."

\section{THE IMPORTANCE OF SYSTEMATIC EXAM- INATIONS OF UTERINE SCRAPINGS AND EXCISED PORTIONS OF THE CERVIX.}

BY PALMER FINDLEY, M.D.

GLINICAL INETRUCTOR OF GYNECOLOGY, NORTHWESTERN UNIVERSITY MRDIC A SCHOOL. cHICAGo.

This paper has for its object: 1, the emphasis of the importance of systematic examinations of uterine scrapings and excised portions as an aid to diagnosis in gynecology; 2, the presentation of a well known method of making such examinations; 3 , the setting forth of its limitations; 4 , the relation of the microscopic to the macroscopic appearances; 5 , the relation of the microscopic findings to the clinical manifestations.

It will be the purpose of the writer to demonstrate that such systematic examinations can be made by all who have a practical knowledge of microscopy; that without such systematic examinations a malignant growth will often escape detection until clinical manifestations point to a growth too far advanced to per mit of a radical removal, and that many times such an examination is the only means of making a positive early diagnosis. It is the custom of the writer to preserve for examination all products of curettage, to make a systematic microscopic examination of them, which is kept on record, and to which subsequent reference can be made. All who have followed this routine method can not fail to appreciate the importance of such examinations. Where a malignant growth has not been suspected it has been detected in its early development; on the other hand, a condition highly suspicious of malignancy has been proven innocent. In the majority of cases the microscope has only served to verify the clinical diagnosis. Not infrequently has a timely hysterectomy been performed as a direct result of the microscopic findings, and, on the contrary, where the clinical symptoms strongly indicated a hysterectomy for a malignant growth the microscopic examination contraindicated and precluded the radical and formidable operation. It is therefore evident that the greater part of the examinations will no more than verify the clinical diagnosis, yet the occasional finding of an unsuspected malignant condition, the verification of a suspicion and the disqualification of a wrong clinical diagnosis will be sufficient reward for the time and labor expended. The practice of making these examinations is becoming more general. The busy surgeon usually trusts the work to an assistant pathologist or colleague, and in most hospitals such examinations are made as a matter of routine by the resident pathologist.

The folowing method adopted by the writer will be presented in the belief that it will be found to be the most reliable and simple method for diagnostic purposes; it is the simple method of practical utility to the general surgeon. Where original research in the histologic and microchemic methods is desired the reader is referred to modern works on microscopy.

Because of the difficulty in obtaining sections of the mucous membrane of the corpus uteri the tissues are best obtained by curettage; while with the cervix the mucous membrane is so closely adherent to the underlying tissues and the desired tissue is so easily excised it is preferable to remove a wedge-shaped piece of the cervix for an examination. The section should include some healthy tissue, in order to make a microscopic field of the entire pathologic condition together with some healthy tissue. Scrapings are never so satisfactory for examination as are excised pieces; the relations of the histoloyic elements are disturbed, the extent of the lesion can never be ascertained, and though the scrapings may show only inflammatory changes the underlying tissue may be the seat of a malignant growth.

\section{CELLOIDIN EMBEDDING METHOD.}

The scrapings and excised pieces are first cleansed of blood in cold water. They are then placed in a 4 per cent. aqueous solution formalin for twelve hours, then in 50 per cent. alcohol for twenty-four hours, next in 70 per cent. alcohol for twenty-four hours, followed by 95 per cent. alcohol for twenty-four hours, and finally hardened in absolute alcohol for twenty-four hours. The specimen may be placed directly in 50 per cent. alcohol without fixing in formalin, and if the sections are small they may be placed directly in 95 per cent. alcohol for twenty-four to forty-eight hours and then in absolute alcohol for twenty-four to forty-eight hours. After the tissue is thoroughly hardened in the alcohol solutions they are placed in a dilute solution of celloidin for twenty-four hours. If the solution is quite dilute it is not necessary to precede this step by placing them in a solution of equal parts of commercial ether and absolute alcohol for twelve to twenty-four hours, thus saving time. The next step is to place them in a thick solution of celloidin for twelve hours, then mount on cork or a block of wood in celloidin, expose the mounted specimen to the open air for a few minutes, then place in 70 per cent. alcohol for a few hours and the specimen is ready for section cutting. The most satisfactory stain is the double stain of hematoxylin and eosin. In this way about eight days are required, but the actual time spent in the work is not so great providing all necessary material is at hand. Where time is an essential factor the paraffin method may be adopted and the specimen ready in forty-eight to seventy-two hours. For serial sections the paraffin method may be used, but for ordinary usage the serious objection to its use is the necessity of an oven for the purpose of keeping the paraffin at the proper temperature. Dr. Cullen of Johns Hopkins University has presented to the profession a freezing method by which the examination can be made in fifty to sixty minutes.

The steps in the method are, in brief: 1 , tissues cut with a freezing microtome; 2 , sections fixed in a 4 per cent. formalin solution, three to five minutes; 3,50 per cent. alcohol, three minutes; 4, absolute alcohol for one minute; 5, stain and mount. By this method it is possible in a doubtful case to determine upon further operative procedures within an hour after the exploratory curettage, and hence is of the greatest value in those exceptional cases where an immediate diagnosis is imperative, otherwise it is not satisfactory because of the hasty and imperfect preparation of the section for microscopic examination.

By way of illustrating the importance of such examinations the writer will briefly mention his

I For a full description of the method see Johns Hopkins Bulletin, No. 49, April, 1895 . 